Research Article

\title{
Causes, evaluation and surgical management of mechanical small bowel obstruction
}

\author{
Md. Shakeb Ahmad', Md. Shadab ${ }^{1}$, Saif Omar ${ }^{2}$, Amjad Zia Mallik', Md. Arif Ansari
}

${ }^{1}$ Department of Surgery, Katihar Medical College, Katihar, Bihar, India
${ }^{2}$ Department of Anatomy, Katihar Medical College, Katihar, Bihar, India

Received: 22 September 2015

Accepted: 07 October 2015

\section{*Correspondence:}

Dr. Saif Omar,

E-mail: drsaifomar@gmail.com

Copyright: ( ) the author(s), publisher and licensee Medip Academy. This is an open-access article distributed under the terms of the Creative Commons Attribution Non-Commercial License, which permits unrestricted non-commercial use, distribution, and reproduction in any medium, provided the original work is properly cited.

\begin{abstract}
Background: Small bowel obstruction is defined as a mechanical or functional obstruction of the intestine preventing the normal transit of product of digestion. It can occur at any level distal to the duodenum and is considered to be a medical emergency. Acute intestinal obstruction has attracted wide attention and extensive research. As a consequence of experimental research, a considerable decline has been observed in the mortality of acute intestinal obstruction. The aim of this study was to observe the causes, evaluation and surgical management of small bowel obstruction among cases presenting with the same in the Department of General Surgery of Katihar Medical College. Methods: Eighty cases of small bowel obstruction were observed in this study. The cases were selected among patients visiting the outdoor clinic of General Surgery in Katihar Medical College. The selected cases belonged to different aetiology, age groups and sex.

Results: Abdominal tenderness was the commonest finding during per abdomen examination. Adhesion was the commonest aetiology observed in this study followed by obstructed hernia. General trend of reporting to the hospital was observed to be on the third day of onset. Higher incidence of small bowel obstruction was observed in cases in sixth decade of life. There was predominance among the males and in cases belonging to lower socio-economic class. Conclusions: Mechanical obstruction of the small intestine due to extrinsic causes constituted a major part among the presenting cases in this study. The degree of distension has a direct relation with the duration of the symptom. Small bowel obstruction can be confirmed using a radiogram in erect posture. Mortality due to small bowel obstruction can be reduced if timely intervention is sought by the presenting cases. Early diagnosis, effective resuscitation and timely surgery are the main treatment options that should be considered.
\end{abstract}

Keywords: Bowel, Diagnosis, Distension, Duodenum, Obstruction, Surgery

\section{INTRODUCTION}

The small intestine consists of the duodenum, jejunum and ileum. It extends from the distal end of the pyloric canal to the ileocaecal valve. Its overall length ranges from three to seven meters in the living adult. The duodenum extends up to the duodenojejunal junction and the remaining small intestine is often referred to as the "small bowel" of which proximal two-fifths is jejunum and distal three-fifths is ileum. ${ }^{1}$ Acute intestinal obstruction occurs when there is an interruption in the forward flow of intestinal contents. This interruption can occur at any point along the length of the gastrointestinal tract, and clinical symptoms often vary based on the level of obstruction. Intestinal obstruction is most commonly caused by intraabdominal adhesions, malignancy, or intestinal herniation. The clinical presentation generally includes nausea and vomiting, colicky abdominal pain, and a failure to pass flatus or bowel movements. The classic physical examination findings of abdominal 
distension, percussion sounds, and high-pitched bowel sounds suggest the diagnosis. Radiologic imaging can confirm the diagnosis, and can also serve as a useful investigation in less certain cases. Although radiography is often the initial study, non-contrast computed tomography is recommended if the index of suspicion is high or if suspicion persists despite negative radiography. Management of uncomplicated obstructions includes fluid resuscitation with correction of metabolic derangements, intestinal decompression, and bowel rest. Evidence of vascular compromise or perforation, or failure to resolve with adequate bowel decompression is an indication for surgical intervention. Intestinal obstruction accounts for approximately 15 percent of all emergency department visits for acute abdominal pain. ${ }^{2}$ Complications of intestinal obstruction include bowel ischemia and perforation. Morbidity and mortality associated with intestinal obstruction have declined since the advent of more sophisticated diagnostic tests, but the condition remains a challenging surgical diagnosis. Physicians who are treating patients with intestinal obstruction must weigh the risks of surgery with the consequences of inappropriate conservative management. The fundamental concerns about intestinal obstruction are its effect on whole body fluid/electrolyte balances and the mechanical effect that increased pressure has on intestinal perfusion. Proximal to the point of obstruction, the intestinal tract dilates as it fills with intestinal secretions and swallowed air. ${ }^{3}$ Failure of intestinal contents to pass through the intestinal tract leads to a cessation of flatus and bowel movements. Intestinal obstruction can be broadly differentiated into small bowel and large bowel obstruction. Fluid loss from emesis, bowel edema, and loss of absorptive capacity leads to dehydration. Emesis leads to loss of gastric potassium, hydrogen, and chloride ions, and significant dehydration stimulates renal proximal tubule reabsorption of bicarbonate and loss of chloride, perpetuating the metabolic alkalosis. ${ }^{4}$ In addition to derangements in fluid and electrolyte balance, intestinal stasis leads to overgrowth of intestinal flora, which may lead to the development of feculent emesis. Additionally, overgrowth of intestinal flora in the small bowel leads to bacterial translocation across the bowel wall..$^{5}$ Persistently increasing dilation of the intestine increases the luminal pressures. When the luminal pressures exceed venous pressures, loss of venous drainage causes increasing edema and hyperaemia of the bowel. This may eventually lead to compromised arterial flow to the bowel, causing ischemia, necrosis, and perforation. A closed loop obstruction, in which a section of bowel is obstructed proximally and distally, may undergo this pro-cess rapidly, with few presenting symptoms. Intestinal volvulus, the prototypical closedloop obstruction, causes torsion of arterial inflow and venous drainage, and is a surgical emergency. The most common causes of intestinal obstruction include adhesions, neoplasms, and herniation. Adhesions resulting from prior abdominal surgery are the predominant cause of small bowel obstruction, accounting for approximately 60 percent of cases. ${ }^{6}$ Lower abdominal surgeries, including appendectomies, colorectal surgery, gynecologic procedures, and hernia repairs, confer a greater risk of adhesive small bowel obstruction. Less common causes of obstruction include intestinal intussusceptions, volvulus, intra-abdominal abscesses, gallstones, and foreign bodies. The development of metabolic acidosis, especially in a patient with an increasing serum lactate level, may signal bowel ischemia.

\section{METHODS}

After prior approval from the Institutional Ethics Committee (IEC). A prospective observational study of eighty cases which were admitted with small bowel obstruction was performed in the Department of General Surgery, Katihar Medical College.

\section{RESULTS}

All eighty cases of small bowel obstruction observed in this study presented with common symptoms of abdominal distension, absence of flatus and/or faeces. Twenty $(25 \%)$ cases were in the sixth decade of life. Preponderance of males over females was observed with fifty-five $(68.75 \%)$ male cases. Fifty-two (65\%) cases were from lower socio-economic strata. The commonest cause was adhesion followed by hernia which were observed in thirty-three $(41.25 \%)$ and twenty $(25 \%)$ of the presenting cases. Thirty-eight $(47.5 \%)$ cases and twenty-two $(27.5 \%)$ cases reported to the hospital on the third day and second day respectively. Degree of abdominal distension was moderate in twenty-five $(31.25 \%)$ cases and mild in twenty-three $(28.75 \%)$ cases. High pitched or abnormal bowel sounds were heard in twenty-seven $(33.75 \%)$ cases. Radiologic investigations revealed multiple fluid levels or gas shadows in fifty-five $(68.75 \%)$ cases. Sonologic investigations revealed small bowel obstruction in twenty-five $(31.25 \%)$ cases. Sixty $(75 \%)$ cases were cured successfully without any complications.

\section{DISCUSSION}

Acute mechanical bowel obstruction is a common surgical emergency and a frequently encountered problem in abdominal surgery. ${ }^{7}$ It constitutes a major cause of morbidity and financial expenditure in hospitals around the world and a significant cause of admissions to emergency surgical departments. ${ }^{8,9}$ Intestinal obstruction belongs to highly severe conditions, requiring a quick and correct diagnosis as well as immediate, rational and effective therapy. ${ }^{10}$ Surgeons are concerned about bowel obstruction cases because strangulation, causing bowel ischemia, necrosis and perforation might be involved, and it is often difficult to distinguish simple obstruction from strangulation. Accurate early recognition of intestinal strangulation in patients with mechanical bowel obstruction is important to decide on emergency surgery or to allow safe nonoperative management of carefully 
selected patients. ${ }^{11}$ Although close and careful clinical evaluation, in conjunction with laboratory and radiologic studies, is essential for the decision of proper management of patients with acute mechanical bowel obstruction, a preoperative diagnosis of bowel strangulation cannot be made or excluded reliably by any known parameter, combinations of parameters, or experienced by clinical judgement. Mechanical bowel obstruction is an old and common surgical emergency. Immediate and correct diagnosis of this condition and its etiology is essential, and appropriate treatment is of utmost importance. $^{12,13}$ The clinical picture, however, of these patients along with the etiology of obstruction and strangulation prevalence are variable, while appropriate management remains controversial. We, therefore, conducted this prospective study to identify and analyse the clinical presentation of patients with acute mechanical bowel obstruction in our department the etiology of obstruction as well as management and outcome of these patients. Age incidence in our study was highest in the sixth decade of life and lowest in the first decade of life respectively. However age is no bar for the acute onset of small bowel obstruction. In early age groups the common causes are congenital bands and intussusceptions whereas adhesions and obstructed hernias are more common in later age groups. Sex ratio revealed that males were more prone to the occurrence of small bowel obstructions. There is no direct relation of intestinal obstruction with sex of the person. A remarkably high incidence was observed among cases belonging to the lower socioeconomic strata. The cases reported to the hospital usually on the third day after development of symptoms. It was observed that prognosis and management among cases which reported earlier was better due to minimal chances of gut injury or strangulation. Degree of abdominal distension has a direct relation with the duration and intensity of the symptom. Abdominal tenderness was observed in thirty-seven cases and eleven cases revealed visible peristalsis. Both mild and moderate distensions were observed. Confirmation of small bowel obstruction was by radiologic and sonologic investigations. Presence of peristalsis helps to differentiate small bowel obstruction from a paralytic ileus. ${ }^{14}$ Management of intestinal obstruction is directed at correcting physiologic derangements caused by the obstruction, bowel rest, and removing the source of obstruction. $^{15}$

\section{CONCLUSION}

Abdominal distension along with absence of passage of flatus and/or faeces is the most common symptoms and physical finding of patients with acute mechanical bowel obstruction. Adhesions and hernias are the most common causes of obstruction as well as of bowel ischemia, necrosis, and perforation. A large number of these patients can be safely and effectively nonoperatively treated, particularly those with adhesive obstruction, a substantial portion requires immediate operation. Moreover, the risk of strangulation is significantly higher in incarcerated hernias than other obstruction causes. Great caution should be taken for the treatment of patients with acute mechanical bowel obstruction since the incidence of bowel ischemia, necrosis, and perforation is significantly high. Further studies are necessary in order to determine appropriate management for treatment of these patients as well as to identify accurate early predictors of success of conservative or operative treatment and, particularly, of intestinal strangulation giving the greatest attention to reversible ischemia.

\section{Funding: No funding sources \\ Conflict of interest: None declared}

Ethical approval: The study was approved by the institutional ethics committee

\section{REFERENCES}

1. Standring S. Gray's Anatomy, 40th Edition: Elsevier Churchill Livingstone. Chapter 66, Small Intestine. 2008:1125.

2. Irvin TT. Abdominal pain: a surgical audit of 1190 emergency admissions. $\mathrm{Br}$ J Surg. 1989;76(11):1121-5.

3. Wright HK, O'Brien JJ, Tilson MD. Water absorption in experimental closed segment obstruction of the ileum in man. Am J Surg. 1971;121(1):96-9.

4. Wangensteen $\mathrm{OH}$. Understanding the bowel obstruction problem. Am J Surg. 1978;135(2):13149.

5. Rana SV, Bhardwaj SB. Small intestinal bacterial over-growth. Scand J Gastroenterol. 2008;43(9):1030-7.

6. Shelton BK. Intestinal obstruction. AACN Clin Issues. 1999;10(4):478-91.

7. Mucha P Jr. Small intestinal obstruction. Surg Clin North Am. 1987;67:597-620.

8. Miller G, Boman J, Shrier I, Gordon PH. Etiology of small bowel obstruction. Am J Surg. 2000;180:33-6.

9. Miller G, Boman J, Shrier I, Gordon PH. Natural history of patients with adhesive small bowel obstruction. Br J Surg. 2000;87:1240-7.

10. Dite P, Lata J, Novotny I. Intestinal obstruction and perforation--the role of the gastroenterologist. Dig Dis. 2003; 21:63-7.

11. Richards WO, Williams LF Jr. Obstruction of the large and small intestine. Surg Clin North Am. 1988;68:355-76.

12. Renzulli P, Krahenbuhl L, Sadowski C, al-Adili F, Maurer CA, Buchler MW. Modern diagnostic strategy in ileus. Zentralbl Chir. 1998;123:1334-9.

13. Lopez-Kostner F, Hool GR, Lavery IC. Management and causes of acute large-bowel obstruction. Surg Clin North Am. 1997;77:1265-90.

14. Frager DH, Baer JW. Distinction between postoperative ileus and mechanical small bowel obstruction: value of CT compared with clinical and 
other radiographic findings. AJR Am J Roentgenol. 1995;164(4):891-4.

15. Markogiannakis $H$. Acute mechanical bowel obstruction: Clinical presentation, etiology, management and outcome. World J Gastroenterol. 2007;13(3):432-7.

Cite this article as: Ahmad MS, Shadab M, Omar S, Mallik AZ, Ansari MA. Causes, evaluation and surgical management of mechanical small bowel obstruction. Int Surg J 2015;2:492-5. 